TRANSACTIONS OF THE

AMERICAN MATHEMATICAL SOCIETY

Volume 354, Number 3, Pages 945-966

S 0002-9947(01)02886-0

Article electronically published on October 24, 2001

\title{
GROUPS DEFINABLE IN SEPARABLY CLOSED FIELDS
}

\author{
E. BOUSCAREN AND F. DELON
}

\begin{abstract}
We consider the groups which are infinitely definable in separably closed fields of finite degree of imperfection. We prove in particular that no new definable groups arise in this way: we show that any group definable in such a field $L$ is definably isomorphic to the group of $L$-rational points of an algebraic group defined over $L$.
\end{abstract}

\section{INTRODUCTION AND PRELIMINARIES}

1.1. Introduction. While looking at questions related to the classification of minimal types in separably closed fields of finite degree of imperfection, we realized that the question of whether a group definable in such a separably closed field $L$ was definably isomorphic to the group of $L$-rational points of an algebraic group defined over $L$. The previous results on the subject [Me 94] stated (Theorem 2.6) that a definable group was definably isomorphic to an " $L$-algebraic group", where an $L$ algebraic group meant a group $H$, definable in $L^{m}$ for some $m$, obtained by gluing together a finite number of Zariski closed subsets of $L^{m}$, with rational charts and locally rational multiplication and inverse, all defined over $L$ (in the present paper, we call these groups $\lambda_{0}$-algebraic groups). Going from an $L$-algebraic group in this (weak) sense to the group of $L$-rational points of an algebraic group defined over $L$, that is, to a group with multiplication locally rational also in the algebraic closure of $L, \bar{L}$, requires the application of a Weil-type theorem on generically defined groups in $\bar{L}$, even if one just wants to obtain an embedding. Furthermore, we then realized that there was a mistake appearing early on in the proof in Me 94]. There are two different notions of generic points for a connected definable group in $L$, one coming from the model-theory of stable groups, the other coming from the topology naturally associated to $L$ (the $\lambda$-topology). These two notions do not necessarily coincide, we give an example in section 5] This had been overlooked in [Me 94, and ever since it seems. The assumption that these two notions of generics coincide (or equivalently that the generic types of a definable group are associated to minimal ideals) was used twice: first in order to prove that every definable group is connected-by-finite (Lemma 2.2) and then (in Proposition 2.5) on the way towards proving that connected definable groups are definably isomorphic to $L$-algebraic groups.

In this paper we answer the original question positively, that is we prove that a definable group in $L$ is isomorphic to the group of $L$-rational points of an algebraic group defined over $L$ and on the way we prove that all definable groups are indeed

Received by the editors January 10, 1999 and, in revised form, September 20, 2000.

1991 Mathematics Subject Classification. Primary 03C60, 03C45, 12L12.

Key words and phrases. Separably closed fields, groups. 
connected-by-finite. We use one result (Proposition 2.4) from [Me 94, which we reprove here as we need it in a context slightly more general than the original one (this is our Lemma 3.1).

We start with two rather detailed preliminaries (Sections 1.2 and 1.3 ) in order to fix the notation and to recall precisely the main model theoretic and algebraic results we will use all the time. One such basic result is the fact that to any complete type over a separably closed field $K$ is associated a prime separable ideal in the ring of polynomials over $K$ in countably many variables. In Section 2 , we prove the existence in this polynomial ring of ideals which are minimal amongst the prime separable ideals containing a given finitely generated ideal. This result which is essential for proving that all definable groups are connected-by-finite, corrects an inaccurate statement (Lemma 46) in De 88. In Section 3 we consider various notions of "local" rationality of the group law. The various possible levels of rationality of the group law turn out, not too surprisingly, to be very relevant to this correspondence between group generics and topological generics. In Section [4 we prove the main results already described above. In the final section (Section 5) we present various examples which emphasize the difficulties one may run into when dealing with a separably closed but non-algebraically closed field.

In a forthcoming paper BoDe] we use the main results of this paper to give a description of minimal infinitely definable groups in separably closed fields of finite degree of imperfection. In particular we answer positively the question of the existence of such a group with infinite transcendence degree (i.e. a minimal group with non-thin generic).

1.2. Preliminaries on separably closed fields. We recall briefly the main features of the model theory of separably closed fields, mainly in order to fix the notation we use. The reader can find more detailed expositions in the following papers: De 88, [Me 96], [De 98. Definitions and previous results on the $\lambda$-topology can be found in Me 94 and De 98.

A field $K$ is said to be separably closed if it has no proper separable algebraic extension. From now on we consider fields of fixed characteristic $p>0$. The index $\left[K: K^{p}\right]$ is either infinite or equal to $p^{\nu}$ for some integer $\nu \geq 0$; in that case, $\nu$ is called the degree of imperfection of $K$. If $\nu \geq 1$, the field $K$ is not algebraically closed and the theory of separably closed fields of finite fixed degree of imperfection $\nu \geq 1$ is complete, stable and not superstable. It also admits quantifier elimination and elimination of imaginaries in a fairly natural language as we will see more precisely a little below.

1.2.1. Some algebra and some notation. Let $K$ be a field of finite degree of imperfection $\nu \geq 1$. A subset $B=\left\{b_{1}, \ldots, b_{\nu}\right\}$ of $K$ is called a $p$-basis of $K$ if the set of p-monomials of $B$,

$$
\left\{M_{j}:=\prod_{i=1}^{\nu} b_{i}{ }^{j(i)} ; j \in p^{\nu}\right\}
$$

(with $p^{\nu}$ identified with the set of maps from $\{1, \ldots, \nu\}$ to $\{0, \ldots, p-1\}$ ), forms a linear basis of $K$ over $K^{p}$. We let $\underline{0}$ denote the constant map with value 0 , i.e. $M_{\underline{0}}=1$. Each element $x$ in $K$ can be written in a unique way as $x=\sum_{j \in p^{\nu}} x_{j}{ }^{p} M_{j}$. 
Note that if $L \supseteq K, B$ remains a $p$-basis for $L$ if and only if $L$ is a separable extension of $K$ and is also of degree of imperfection $\nu$.

From now on we fix a p-basis $B$ of $K$ and the $M_{j}$ 's, with $j \in p^{\nu}$, always denote the p-monomials of $B$.

We let $f_{j}$ denote the map which to $x$ associates $x_{j}$. The $x_{j}$ 's are called the $p$-components of $x$ of level one. More generally, one can associate to $x$ a tree of countable height indexed by $\left(p^{\nu}\right)^{<\omega}$, which we call the tree of $p$-components of $x$. For $\sigma \in\left(p^{\nu}\right)^{<\omega}$, we define $x_{\sigma}$ by induction: $x_{\emptyset}=x$ and if $\tau \in\left(p^{\nu}\right)^{n}$, and $j \in p^{\nu}$, we let $x_{(\tau, j)}$ be equal to $f_{j}\left(x_{\tau}\right) ; x_{(\tau, j)}$ is called a $p$-component of $x$ of level $n+1$. We define also, for $\sigma \in\left(p^{\nu}\right)^{<\omega}$, the $f_{\sigma}$ 's, the iterated p-component maps, by setting $f_{\sigma}(x)=x_{\sigma}$ or, equivalently, that $f_{\emptyset}$ is the identity and that if $\tau \in\left(p^{\nu}\right)^{n}$ and $j \in p^{\nu}$, then $f_{(\tau, j)}:=f_{j} \circ f_{\tau}$.

One can also define in a similar way the iterated $p$-monomials of $B$ : for $\tau \in\left(p^{\nu}\right)^{n}$, $n \geq 1$, and $j \in p^{\nu}$, let

$$
M_{(\tau, j)}=M_{\tau} M_{j}^{p^{n}}
$$

We then have, for each $n \geq 1$,

$$
x=\sum_{\sigma \in\left(p^{\nu}\right)^{n}} x_{\sigma}^{p^{n}} M_{\sigma} .
$$

We will also need, for each $n \geq 0$, maps $\lambda_{n}$ which to $x \in K$ associate the tuple of its $p$-components of level $n$ : $\lambda_{n}$ is a map from $K$ to $K^{\times p^{\nu n}}$ (the cartesian product of $p^{\nu n}$ copies of $\left.L\right)$, defined by $\lambda_{n}(x):=\left(x_{\sigma}\right)_{\sigma \in\left(p^{\nu}\right)^{n}}$. We extend this notation to elements of $K^{\times k}$ : for $a=\left(b_{1}, \ldots, b_{k}\right) \in K^{\times k}$ and $\sigma \in\left(p^{\nu}\right)^{n}, a_{\sigma}$ will denote the $k$-tuple $\left(\left(b_{1}\right)_{\sigma}, \ldots,\left(b_{k}\right)_{\sigma}\right) \in K^{\times k}$ and $\lambda_{n}(a)$ the tuple $\left(a_{\sigma}\right)_{\sigma \in\left(p^{\nu}\right)^{n}} \in K^{\times k p^{\nu n}}$. We will also use the following abbreviations: $a_{\leq n}$ for the tuple $\left(a_{\sigma}\right)_{\sigma \in\left(p^{\nu}\right) \leq n}$ and $a_{\infty}$ for the infinite sequence $\left(a_{\sigma}\right)_{\sigma \in\left(p^{\nu}\right)<\omega}$.

Separability and ideals. Let $C$ be a commutative $K$-algebra.

An ideal $I$ of $C$ is separable if, for all $c_{j} \in C, j \in p^{\nu}$,

$$
\text { if } \sum_{j \in p^{\nu}} c_{j}^{p} M_{j} \in I, \text { then each } c_{j} \in I .
$$

Facts. (i) A prime ideal $I$ of $C$ is separable iff the quotient field of $C / I$ is a separable extension of $K$.

(ii) Let $Q, J$ be ideals of $C$. Suppose that $Q \cap J$ is separable, $J \nsubseteq Q$ and $Q$ is prime. Then $Q$ is separable.

(iii) A separable ideal of $C$ is radical.

The ring $K\left[X_{\infty}\right] . K\left[X_{\infty}\right]$ is the polynomial ring in countably many indeterminates indexed in a way which will allow the natural substitution by the $p$-components of elements: for $X$ a single variable, $X_{\infty}:=\left(X_{\sigma}\right)_{\sigma \in\left(p^{\nu}\right)<\omega}$, and for $X=\left(Y_{1}, \ldots, Y_{k}\right)$ a $k$-tuple of variables, $X_{\infty}:=\left(\left(Y_{1}\right)_{\infty}, \ldots,\left(Y_{k}\right)_{\infty}\right)$. The ring $K\left[X_{\infty}\right]$ is a countable union of Noetherian rings, hence each ideal is countably generated.

1. For $X$ a single indeterminate $I^{0}(X)$ is the ideal of $K\left[X_{\infty}\right]$ generated by the polynomials $X_{\sigma}-\sum_{j \in p^{\nu}} X_{(\sigma, j)}^{p} M_{j}, \sigma \in\left(p^{\nu}\right)^{<\omega}$. If $X=\left(X_{1}, \ldots, X_{k}\right)$ is a $k$ tuple of indeterminates and if there is no risk of confusion, $I^{0}(X)$ denotes the ideal generated by $\sum_{i=1}^{k} I^{0}\left(X_{i}\right)$. By abuse of notation and again if there is no risk of confusion, we also denote by $I^{0}(X)$ the ideal generated by $I^{0}(X)$ in $K\left[X_{\infty}, Y_{\infty}\right]$. 
2. Define, for $X$ a single variable, $X_{\leq n}:=\left(X_{\sigma}\right)_{\sigma \in\left(p^{\nu}\right) \leq n}$ and for $X=\left(Y_{1}, \ldots, Y_{k}\right)$ a $k$-tuple of variables, $X_{\leq n}:=\left(\left(Y_{1}\right)_{\leq n}, \ldots,\left(Y_{k}\right)_{\leq n}\right)$. Then, for an ideal $I$ of $K\left[X_{\infty}\right]$, $I_{\leq n}:=I \cap K\left[X_{\leq n}\right]$. Clearly $I$ is separable (or prime) iff each $I_{\leq n}$ is.

3. Let $L$ be a separable extension of $K$ of degree of imperfection $\nu$. For $a \in L^{\times k}$, we let $I(a):=\left\{f \in K\left[X_{\infty}\right] ; f\left(a_{\infty}\right)=0\right\}$. Note that $I(a)$ is separable and contains $I^{0}(X)$.

\subsubsection{Model theory and topology.}

Fact 1.1. 1. The theory of separably closed fields of characteristic $p$, of degree of imperfection $\nu$, and with $p$-basis $\left\{b_{1}, \ldots, b_{\nu}\right\}$ admits elimination of quantifiers and elimination of imaginaries in the language

$$
\mathcal{L}_{p, \nu}=\{0,1,+,-, .\} \cup\left\{b_{1}, \ldots, b_{\nu}\right\} \cup\left\{f_{i} ; i \in p^{\nu}\right\} .
$$

In fact a term of $\mathcal{L}_{p, \nu}$ in $x_{1}, \ldots, x_{k}$ is, modulo this theory, equivalent to a polynomial in $\lambda_{n}\left(x_{1}\right), \ldots, \lambda_{n}\left(x_{k}\right)$, for some integer $n$.

2. Let $K \preceq L$ be two models, then for any $a \in L^{\times k}$, the definable closure of $K \cup\{a\}$ is the field $K\left(a_{\infty}\right)$ and the algebraic closure of $K \cup\{a\}$ is the separable closure of $K\left(a_{\infty}\right)$, which is also the prime model over $K \cup\{a\}$ and which we denote by $K\langle a\rangle$.

3. For all $a, b \in L^{\times k}$ and all $n \geq 0$, if $a \in K(b)$, then $\lambda_{n}(a) \in K\left(\lambda_{n}(b)\right)$.

From now on, we do not differentiate, unless we have a special reason, between elements and finite tuples, so when we write $a \in K$, we mean that $a$ is a tuple of elements from some $K^{\times k}$.

From now on we fix $K$, a separably closed field of finite degree of imperfection $\nu$, which is $\left(2^{\aleph_{0}}\right)^{+}$-saturated, and a $p$-basis $B=\left\{b_{1}, \ldots, b_{\nu}\right\}$ of $K$. We also fix $L$, separably closed of the same degree $\nu$, which is an elementary extension of $K$ (that is a separable extension) and which is $|K|^{+}$-saturated.

Fact 1.2. For $a \in L^{\times k}$, the map $a \rightarrow I(a)$ induces a bijection between complete $k$-types over $K$ and prime separable ideals $I$ of $K\left[X_{\infty}\right]$ containing $I^{0}(X)$. The ideal $I^{0}(X)$ (in one single variable) corresponds to the generic type of the field $L$. Tuples $a$ and $d$ from $L$ are independent over $K$ iff $K\langle a\rangle$ and $K\langle d\rangle$ are linearly (or equivalently algebraically) disjoint over $K$.

Because of this one-to-one correspondence we call prime separable ideals of $K\left[X_{\infty}\right]$ which contain $I^{0}(X)$ type ideals.

We now recall the definition of the $\lambda$-topology:

1. Given a set of polynomials $S$ of $K\left[X_{\infty}\right]$, we define

$$
V(S)=\left\{a \in L^{\times k} ; f\left(a_{\infty}\right)=0 \text { for all } f \in S\right\} .
$$

Such a $V(S)$ is called $\lambda$-closed (with parameters in $K$ or over $K$ ) in $L$.

2. Given $A \subseteq L^{\times k}$, we define its canonical ideal $I(A)$,

$$
I(A):=\left\{f \in K\left[X_{\infty}\right] ; f\left(a_{\infty}\right)=0 \text { for all } a \in A\right\} .
$$

The $\lambda$-closed subsets of $L^{\times k}$ form the closed sets of the $\lambda$-topology on $L^{\times k}$. In this topology $V(I(A))$ is the closure of $A$, its $\lambda$-closure. As usual a closed set is called irreducible, or $\lambda$-irreducible if it is not the proper union of two closed subsets and a maximal irreducible closed subset of some closed set $A$ is called an irreducible component of $A$. A point of some irreducible closed subset $A$ is called a topological generic of $A$ if its closure is $A$. Each irreducible closed subset is contained in an 
irreducible component. If a closed set $A$ is the union of finitely many maximal irreducible closed sets $A_{i}$, then the $A_{i}$ 's are all the irreducible components of $A$.

Fact 1.3 ("Nullstellensatz"). 1. The map $A \rightarrow I(A)$ induces a bijection between $\lambda$-closed subsets of the affine space $L^{\times k}$ with parameters in $K$, and ideals of $K\left[X_{\infty}\right]$ which are separable and contain $I^{0}(X)$. The inverse map is $I \rightarrow V(I)$.

2. A $\lambda$-closed set $A$ is irreducible iff $I(A)$ is prime.

3. Let $C \subseteq A$ be $\lambda$-closed sets. Then $C$ is an irreducible component of $A$ iff $I(C)$ is a prime ideal minimal over $I(A)$.

Remark. By 2 if $A \subseteq L^{\times k}$ is an irreducible $\lambda$-closed set over $K, I(A)$ is prime and there is some complete $k$-type $t$ over $K$ such that $I(t)=I(A)$. The topologically generic points of $A$ are exactly the realizations of the type $t$.

Definition. A set of the form $V(I)$ for a finitely generated $I$ is called $\lambda$-closed of finite type (in [Me 94] such sets are called basic $\lambda$-closed).

Recall that a subset of $L^{\times k}$ is said to be infinitely definable (denoted $M$-definable) if it is an intersection of at most $|K|$ definable subsets.

Any $\lambda$-closed set is a countable intersection of $\lambda$-closed sets of finite type:

$$
x \in V(I) \text { iff } \mathbb{M}_{n} x \in V\left(I_{\leq n}\right)
$$

hence is $M$-definable. By compactness, a $\lambda$-closed set is definable if and only if it is of finite type. In other words, quantifier elimination can be restated as follows: the definable sets are exactly the $\lambda$-constructible sets, that is the finite Boolean combinations of $\lambda$-closed sets of finite type.

1.2.3. Varieties, switching between a separably closed field and its algebraic closure. Let $\bar{L}$ denote the algebraic closure of $L$. We consider abstract varieties defined over $\bar{L}$ as given by a finite number of affine charts glued together via rational maps. By elimination of imaginaries, this enables us to view a variety defined over $\bar{L}$ as a definable object in $\bar{L}$ (see for example [Po 87] or [Pi 98]). Recall that if $V$ is an affine variety in $\bar{L}, V$ is said to be defined over a subfield $k \subseteq \bar{L}$ if the ideal of $V$, $I(V) \subseteq \bar{L}[X]$ is generated by polynomials in $k[X]$. An abstract variety is defined over $k$ if its associated affine charts and rational maps are defined over $k$.

It is well-known that if the field $k$ is not perfect, the affine variety $V$ may very well be definable with parameters from $k$ in the sense of model theory without being defined over $k$ in the sense of algebraic geometry. In order to avoid confusion, we will use the words "defined over" only in the algebraic geometry sense. When talking about model theoretic definability, we will say that a set is definable with parameters in $k$.

If we have a variety $V$ defined over $L$, we can also consider $V(L)$, the set of $L$-rational points of $V$, as a definable set in $L$.

For the basic definitions and properties of varieties and algebraic groups which will be used, see [Pi 98] or Po 87] for a presentation with model theoretic flavor, or more classically La 58].

If $K$ is separably closed, a field extension $K \subseteq F$ is separable iff $\bar{K}$ (the algebraic closure of $K$ ) and $F$ are linearly disjoint over $K$. It follows that any prime separable ideal in $K[X]$ is absolutely prime and any separable ideal in $K[X]$ absolutely reduced i.e. if $J_{1}, \ldots, J_{n}$ are the minimal prime (separable) ideals of $K[X]$ containing a separable ideal $J$, then in $\bar{K}[X]$, the minimal prime ideals containing $J \otimes \bar{K}[X]$ are exactly the $J_{i} \otimes \bar{K}[X]$ 's for $1 \leq i \leq n$. 
Let $J$ be a prime separable ideal in $K[X]$. Consider $V_{0}=V(J \otimes \bar{K})$ in $\bar{L}$. As $J$ is absolutely prime, $V_{0}$ is an irreducible affine variety, which is of course defined over $K$.

If $J$ is a separable ideal in $K[X]$, then by absolute reducibility, the Zariski closed set $V(J \otimes \bar{K})$ and all its irreducible components are defined over $K$.

Finally recall that for any irreducible variety $V$ defined over $K$ separably closed, $V(K)$ is Zariski dense in $V(\bar{K})$.

Notation. We will very often switch, as we just did, between the theory of separably closed fields and the theory of algebraically closed fields, i.e. given some tuple $a \in L$ consider its type over $K$ in the separably closed field $L$, called the (scf)-type, or its type over $K$ in the algebraically closed field $\bar{L}$, called the (acf)-type. In order to avoid confusion and at the risk of redundancy, we use the following notation: let $a \in L^{\times k}$

- the usual notation $t(a / K)$ denotes the (scf)-type of $a$ and $I(a)$ (or $I(q)$ if $q=t(a / K))$ the prime separable ideal associated to it in $K\left[X_{\infty}\right]$,

- the notation $t_{a c f}(a / K)$ denotes the (acf)-type of $a$ considered as a tuple in $\bar{L}$ and $I_{a c f}(a)$ (or $I_{a c f}(t)$ if $\left.t=t_{a c f}(a / K)\right)$ the prime ideal in $K[X]$ associated to it.

1.2.4. The $\Lambda$ functor. We give here a very brief presentation of the natural functors associated to the maps $\lambda_{n}$, which were introduced by Hrushovski in [Hr 96] in the case of algebraic groups. We define a functor $\Lambda$ from the category of varieties defined over $K$ to itself. In the case of an algebraic group defined over $K$, one can check that $\Lambda$ is equal to the composition of the inverse of the Frobenius and of the classical Weil restriction of scalars functor from $K^{1 / p}$ to $K$ (see Sp 98, in particular Exercise 11.4.20 (4)(a)). In the general case of an abstract variety, one must slightly modify the usual definition of the Weil restriction of scalars. Here we give a more direct definition of $\Lambda$, specific to the separably closed fields context.

Let $A$ be a quasi-affine variety defined over $K$, say $A=\bar{A} \backslash V(J)$, with $\bar{A}$ defined over $K$ and $J$ some ideal in $K[X]$. Then we let

$$
\Lambda A=\overline{\lambda(A(L))} \backslash V\left(J\left(\sum_{i \in p^{\nu}} X_{i}^{p} M_{i}\right)\right),
$$

and

$$
\begin{gathered}
\rho_{A}: \Lambda A \mapsto A, \\
\left(x_{i}\right)_{i \in p^{\nu}} \mapsto \sum_{i \in p^{\nu}} x_{i}^{p} M_{i} .
\end{gathered}
$$

One can check that if $f \in K(X)$, there exists a unique $f^{*}=\left(f_{j}^{*}\right)_{j \in p^{\nu}}$, where $f_{j}^{*} \in K\left(X_{i} ; i \in p^{\nu}\right)$, such that

$$
f\left(\sum_{i \in p^{\nu}} X_{i}^{p} M_{i}\right)=\sum_{j \in p^{\nu}}\left(f_{j}^{*}\left(\left(X_{i}\right)_{i \in p^{\nu}}\right)\right)^{p} M_{j}
$$

Then for $h=\left(h_{1}, \ldots, h_{l}\right)$ a rational map defined everywhere on $A$, we let $\Lambda h$ be equal to $\left(h_{1}^{*}, \ldots, h_{l}^{*}\right)$.

One then extends the definitions of $\Lambda$ and $\rho$ to abstract varieties and obtains:

Proposition 1.4. $\quad$ 1. $\Lambda$ is a functor from the category of varieties defined over $K$ to itself. It maps a point to a point, a subvariety to a subvariety, an algebraic group to an algebraic group, and a morphism of an algebraic group 
to a morphism of an algebraic group. Given $A$, a variety defined over $K, \rho_{A}$ is a surjective morphism from $\Lambda A$ onto $A$, and, for any morphism between two varieties $f: A \rightarrow B, f \circ \rho_{A}=\rho_{B} \circ \Lambda f$.

2. For any variety $A$ defined over $K$, and for any separably closed field $L \succeq K$, the restriction of $\rho_{A}$ to $L$-rational points of $\Lambda A$,

$$
\left(\rho_{A}\right)_{\mid L}:(\Lambda A)(L) \mapsto A(L)
$$

is bijective with inverse the map $\lambda_{A}$ obtained by gluing together the $\lambda$ 's on affine charts of $A$.

We let $\Lambda_{1}=\Lambda$ and for $n \geq 1, \Lambda_{n+1}=\Lambda_{n} \circ \Lambda_{1}$.

Note that the functors $\Lambda_{n}$ do not preserve irreducibility: if $A$ is an irreducible Zariski closed set, then $\Lambda_{n} A$ is irreducible for all $n$ iff $A(L)$ is irreducible as a $\lambda$ closed set. The first example in section 5 presents an irreducible Zariski closed set $A$ such that $A(L)$ is not irreducible. On the contrary, if $G$ is an irreducible (i.e. connected) algebraic group, we will see that $G(L)$ is connected (Lemma 4.4), hence it is $\lambda$-irreducible (see 3.5 below) and it follows that $\Lambda_{n} G$ is also irreducible.

1.3. Stable groups. We assume the reader is familiar with the basic facts about the model theory of (infinitely definable) stable groups (generics, connected component, ...). Definitions and results can be found in Po 87, [Pi 96] or [Wa 97].

Let us just recall that an $\mathrm{M}$-definable stable group is said to be connected if it has no proper relatively definable subgroup of finite index, and is said to be connectedby-finite if it has a relatively definable connected subgroup of finite index.

We will need the following two lemmas, which although part of well-known folklore about stable groups, do not seem to be actually stated anywhere in the exact form we need.

Lemma 1.5. Let $T$ be a stable theory, $G$ and $H$ two $\mathrm{X}$-definable connected groups in $T$. Let $p$ and $q$ denote the respective generic types of $G$ and $H$. Suppose that there is a definable map $f$ such that:

- $f$ takes bijectively the type $p$ to the type $q$

- if $a, b$ are independent realizations of $p$, then $f(a . b)=f(a) . f(b)$.

Then $f$ restricted to the type $p$ can be extended to a definable isomorphism from $G$ to $H$.

Proof. For $x \in G$, define $g(x)=f(a) \cdot f\left(a^{-1} x\right)$ for some (any) $a$ generic independent from $x$.

Lemma 1.6. Let $T$ be a stable theory, $M \preceq N, N$ a big saturated model of $T$ and $H$ an $\mathrm{M}$-definable group definable with parameters from $M$. Let $q$ be a type in $H$ over $M$. Then $q$ is the generic of a (unique) connected $M$-definable subgroup of $H$ (definable with parameters from $M$ ) iff $q$ is closed by generic multiplication, i.e. if $a, b$ realize $q$ and are independent over $M$, then a.b realizes the unique non-forking extension of $q$ over $M a$ and over $M b$.

Proof. The conditions imply that the type $q$ must be the unique generic of its stabilizer in $H$.

Finally, recall that by elimination of imaginaries, any group interpretable in a separably closed field of finite invariant is definably isomorphic to a definable group. 


\section{Minimal ideals}

In this section we prove that amongst the type ideals with given trace on $K\left[X_{\leq n}\right]$, there is a unique minimal one. This result corrects the inaccurate statement of Lemma 46 in [De 88] (see counterexample 5.1).

Lemma 2.1. Let $J$ be a prime separable ideal in $K[X]$. Then there are a polynomial $D \in K[X] \backslash J$ and a type ideal $Q \subseteq K\left[X_{\infty}\right]$ such that $Q$ is the smallest type ideal containing $J$ and not $D$. The ideal $Q$ is also the smallest type ideal such that $Q \cap K[X]=J$.

Proof. The multi-variable $X$ is equal to $\left(X_{1}, \ldots, X_{k}\right)$ for some $k \geq 1$. By separability of $J$ the quotient field $K_{1}$ of $K\left[X_{1}, \ldots, X_{k}\right] / J$ is a separable extension of $K$. By saturation of $L$, we can suppose that $K \subseteq K_{1} \subset L$. Let $e \in K_{1}^{\times k}$ be such that $J=\left\{f \in K\left[X_{1}, \ldots, X_{k}\right] ; f(e)=0\right\}$. Choose a separating transcendence basis of $e$ over $K$. To simplify notation we suppose that $e=\left(e_{1}, \ldots, e_{r}, e_{r+1}, \ldots, e_{k}\right)$, for some $r, 1 \leq r \leq k$, where $e_{1}, \ldots, e_{r}$ is the separating basis.

Let

$$
U:=K\left[\left(X_{1}\right)_{\infty}, \ldots,\left(X_{r}\right)_{\infty}, X_{r+1} \ldots, X_{k}\right]
$$

and

$$
S:=\sum_{s=1}^{r} I^{0}\left(X_{s}\right)+J U .
$$

If $R$ is any type ideal in $K\left[\left(X_{1}\right)_{\infty}, \ldots,\left(X_{r}\right)_{\infty},\left(X_{r+1}\right)_{\infty}, \ldots,\left(X_{k}\right)_{\infty}\right]$ which contains $J$, then $R \cap U$ must contain $S$. We are going to show that there is a type ideal $Q$ such that $Q \cap U=S$ and then that this ideal $Q$ is unique and is included in any other type ideal $R$ such that $R_{=0}:=R \cap K\left[X_{1}, \ldots, X_{k}\right]=J$.

Claim 1 (Existence). There exists $c \in L^{\times k}$ such that $I(c) \cap U=S$.

Proof of the claim. Let $c_{1}, \ldots, c_{r}$ in $L$ be independent realizations of the generic type of $L$ over $K$. This means that

$$
I\left(c_{1}, \ldots, c_{r}\right)=\sum_{s=1}^{r} I^{0}\left(X_{s}\right)
$$

and $K\left(c_{1}, \ldots, c_{r}\right)$ and $K\left(e_{1}, \ldots, e_{r}\right)$ are isomorphic over $K$. Now choose $c_{r+1}, \ldots$, $c_{k}$ in the separable closure of $K\left(c_{1}, \ldots, c_{r}\right)$ such that $K\left[c_{1}, \ldots, c_{r}, c_{r+1}, \ldots, c_{k}\right]$ and $K\left[e_{1}, \ldots, e_{r}, e_{r+1}, \ldots, e_{k}\right]$ are isomorphic over $K$.

For every integer $N, K\left(c_{1}, \ldots, c_{r}, c_{r+1}, \ldots, c_{k}\right)$ and $K\left(\left(c_{1}\right)_{\leq N}, \ldots,\left(c_{r}\right)_{\leq N}\right)$ are linearly disjoint over $K\left(c_{1}, \ldots, c_{r}\right)$ as the first field is a separable algebraic extension of $K\left(c_{1}, \ldots, c_{r}\right)$ and the second one the composition of a purely transcendental extension followed by a purely inseparable extension.

Let $f \in I\left(c_{1}, \ldots, c_{r}, c_{r+1}, \ldots, c_{k}\right) \cap U$. This means that for some $N$,

$$
f \in I\left(c_{1}, \ldots, c_{r}, c_{r+1}, \ldots, c_{k}\right) \cap K\left[\left(X_{1}\right)_{\leq N}, \ldots,\left(X_{r}\right)_{\leq N}, X_{r+1}, \ldots, X_{k}\right] .
$$

For every $i, 1 \leq i \leq r$, and every $\tau \in\left(p^{\nu}\right)^{m}, 1 \leq m<N$, we replace in $f$ each $\left(X_{i}\right)_{\tau}$, by its expression, modulo $I^{0}\left(X_{i}\right)$, in terms of $\left(X_{i}\right)_{=N}:=\left(\left(X_{i}\right)_{\sigma}\right)_{\sigma \in\left(p^{\nu}\right)^{N}}$. Then we divide successively, for $i=1, \ldots, r$, by

$$
\left(\left(X_{i}\right)_{\underline{0}}\right)^{p^{N}}-\left(X_{i}-\sum_{\substack{\sigma \in\left(p^{\nu}\right)^{N} \\ \sigma \neq \underline{0}}}\left(\left(X_{i}\right)_{\sigma}\right)^{p^{N}} M_{\sigma}\right) .
$$


We obtain at the end another polynomial $g \in K\left[X_{1}, \ldots, X_{k},\left(X_{1}\right)_{=N}, \ldots,\left(X_{r}\right)_{=N}\right]$ which is congruent to $f$ modulo $\sum_{s=1}^{r} I^{0}\left(X_{s}\right)$ and has degree strictly less than $p^{N}$ in each $\left(X_{i}\right)_{\underline{0}}$. This polynomial can be written as

$$
g=\sum_{j} f_{j}\left(X_{1}, \ldots, X_{k}\right) \Pi_{j}\left(\left(X_{1}\right)_{=N}, \ldots,\left(X_{r}\right)_{=N}\right)
$$

where each $f_{j} \in K\left[X_{1}, \ldots, X_{k}\right]$ and the $\Pi_{j}$ 's are distinct monomials in the variables $\left(X_{i}\right)_{=N}$ of degree strictly less than $p^{N}$ in each $\left(X_{i}\right)_{\underline{0}}$.

The $\Pi_{j}\left(\left(c_{1}\right)_{=N}, \ldots,\left(c_{r}\right)_{=N}\right)$ 's are linearly independent over $K\left(c_{1}, \ldots, c_{r}\right)$, hence over $K\left(c_{1}, \ldots, c_{r}, c_{r+1}, \ldots, c_{k}\right)$ by linear disjointness. This implies that the $f_{j}\left(c_{1}, \ldots, c_{k}\right)$ 's must be equal to zero, i.e. that $f_{j} \in J$. It follows that

$$
f \in \sum_{s=1}^{r} I^{0}\left(X_{s}\right)+J U
$$

and this finishes the claim.

Claim 2 (Minimality). There is a unique type ideal

$$
Q \subseteq K\left[\left(X_{1}\right)_{\infty}, \ldots,\left(X_{r}\right)_{\infty},\left(X_{r+1}\right)_{\infty}, \ldots,\left(X_{k}\right)_{\infty}\right]
$$

such that $Q \cap U=S$. There is a polynomial $D \in K\left[X_{1}, \ldots, X_{k}\right] \backslash J$ such that if $R$ is any type ideal with $R_{=0} \supseteq J$ and $D \notin R$, then $R \supseteq Q$.

Proof of the claim. Let $e \in L^{\times k}$ as above be such that $J=\left\{f \in K\left[X_{1}, \ldots, X_{k}\right]\right.$; $f(e)=0\}$. Consider any $i, r+1 \leq i \leq k$. Then $e_{i}$ is separably algebraic over $e_{1}, \ldots, e_{r}$ and hence $e_{i} \in K\left(e_{1}, \ldots, e_{r}\right)\left[e_{i}^{p}\right]$. It follows that there is $D_{i} \in$ $K\left[X_{1}, \ldots, X_{r}\right] \backslash J$ such that $e_{i} \in K\left[e_{1}, \ldots, e_{r}, e_{i}^{p},\left(D_{i}\left(e_{1}, \ldots, e_{r}\right)\right)^{-1}\right]$ and by induction on $m$, for every integer $m$,

$$
e_{i} \in K\left[e_{1}, \ldots, e_{r}, e_{i} p^{m},\left(D_{i}\left(e_{1}, \ldots, e_{r}\right)\right)^{-1}\right] .
$$

It follows that, for every $m$, there is some integer $u_{i m}$ and some polynomial $G_{i m} \in$ $K\left[X_{1}, \ldots, X_{r}, X_{i}^{p^{m}}\right]$ such that the polynomial $X_{i} D_{i}^{p^{m} u_{i m}}-G_{i m}$ takes value zero on $e$ and hence belongs to the ideal $J$.

We can decompose

$$
\begin{gathered}
X_{i} \equiv \sum_{\sigma \in\left(p^{\nu}\right)^{m}}\left(\left(X_{i}\right)_{\sigma}\right)^{p^{m}} M_{\sigma}\left(\bmod \sum_{s=1}^{k} I^{0}\left(X_{s}\right)\right), \\
G_{i m} \equiv \sum_{\sigma \in\left(p^{\nu}\right)^{m}} G_{i \sigma} p^{m} M_{\sigma}\left(\bmod \sum_{s=1}^{k} I^{0}\left(X_{s}\right)\right),
\end{gathered}
$$

where $G_{i \sigma} \in K\left[\left(X_{1}\right)_{\leq m}, \ldots,\left(X_{r}\right)_{\leq m}, X_{i}\right]$.

If $R \subseteq K\left[\left(X_{1}\right)_{\infty}, \ldots,\left(X_{r}\right)_{\infty},\left(X_{r+1}\right)_{\infty}, \ldots,\left(X_{k}\right)_{\infty}\right]$ is a separable ideal containing $J$ and $\sum_{s=1}^{k} I^{0}\left(X_{s}\right)$, it must contain all the $\left(X_{i}\right)_{\sigma} D_{i}^{u_{i m}}-G_{i \sigma}$ for $r+1 \leq$ $i \leq k, \sigma \in\left(p^{\nu}\right)^{m}$. Suppose furthermore that $R$ is also prime and does not contain $D:=\prod_{i=r+1}^{k} D_{i}$. Let $a \in L^{\times k}$ be such that $I(a)=R$ and $c \in L^{\times k}$ be such that $I(c)=Q$. As $R \cap U \supseteq S=Q \cap U$, there is a homomorphism $h: K\left[\left(c_{1}\right)_{\infty}, \ldots,\left(c_{r}\right)_{\infty}, c_{r+1}, \ldots, c_{k}\right] \mapsto K\left[\left(a_{1}\right)_{\infty}, \ldots,\left(a_{r}\right)_{\infty}, a_{r+1}, \ldots, a_{k}\right]$ with kernel equal to $R \cap U / S$.

As $R$ does not contain $D$ and contains all the $\left(X_{i}\right)_{\sigma} D_{i}^{u_{i m}}-G_{i \sigma}$ for $r+1 \leq i \leq k$, $h$ extends, in a unique way, to a homomorphism from $K\left[\left(c_{1}\right)_{\infty}, \ldots,\left(c_{r}\right)_{\infty},\left(c_{r+1}\right)_{\infty}\right.$, 
$\left.\ldots,\left(c_{k}\right)_{\infty}\right]$ to $K\left[\left(a_{1}\right)_{\infty}, \ldots,\left(a_{r}\right)_{\infty},\left(a_{r+1}\right)_{\infty}, \ldots,\left(a_{k}\right)_{\infty}\right]$. It follows that $Q \subseteq R$ and that $Q$ is the unique type ideal such that $Q \cap U=S$.

Proposition 2.2. Let $J \subseteq K\left[X_{\leq n}\right]$ be a prime separable ideal containing $I^{0}(X)_{\leq n}$. Then there is a type ideal $Q$ in $K\left[X_{\infty}\right]$ which is the smallest type ideal such that $Q_{\leq n}=J$.

Furthermore, there is a polynomial $D \in K\left[X_{\leq n}\right] \backslash J$ such that $Q$ is also the smallest type ideal containing $J$ and not $D$.

This ideal $Q$ will be called the minimal closure of $J$, and denoted $M \operatorname{in}(J)$.

Proof. If $X=\left(X_{1}, \ldots, X_{k}\right)$, we define the multivariable $Y$ of length $k p^{\nu n}$ :

$$
Y=\left(\left(X_{1}\right)_{\sigma}, \ldots,\left(X_{k}\right)_{\sigma}\right)_{\sigma \in\left(p^{\nu}\right)^{n}},
$$

and apply the previous lemma to the ideal $J \cap K[Y]$ in $K[Y]$. Let $Q$ be the ideal of $K\left[Y_{\infty}\right]$ and $D$ the polynomial in $K[Y] \backslash J$ given by the lemma. Let $a \in L^{\times k p^{\nu n}}$ be such that $I(a)=Q, a=\left(\left(a_{1}\right)_{\sigma}, \ldots,\left(a_{k}\right)_{\sigma}\right)_{\sigma \in\left(p^{\nu}\right)^{n}}$. Consider $b \in L^{\times k p^{\nu}}$,

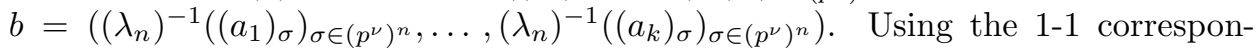
dence between ideals of $K[Y]$ and ideals of $K\left[\left(X_{1}\right)_{\leq n}, \ldots,\left(X_{k}\right)_{\leq n}\right]$ which contain $I^{0}\left(X_{1}, \ldots, X_{k}\right)_{\leq n}$ and the 1-1 correspondence between type ideals in $K\left[Y_{\infty}\right]$ and type ideals of $K\left[\left(X_{1}\right)_{\infty}, \ldots,\left(X_{k}\right)_{\infty}\right]$ induced by the map $\lambda_{n}$, it is easy to see that the ideal $I(b)$ in $K\left[\left(X_{1}\right)_{\infty}, \ldots,\left(X_{k}\right)_{\infty}\right]$ and the polynomial $D$ satisfy the requirements.

We now derive some first consequences of the existence of the minimal closure:

Definition. Let $E \subseteq L^{\times k}$ be an infinitely definable set, definable with parameters from $K$. We define the transcendence degree of $E, \operatorname{trdg}(E)$, to be the maximum of $\{\operatorname{trdg}(K(a): K) ; a \in E\}$. For $n \geq 0$, we denote $E_{\leq n}$ the set $\left\{a_{\leq n} ; a \in E\right\}$.

Note that if $E \subseteq L^{\times k}$, then $\operatorname{trdg}(E)$ is equal to the dimension of the ideal $I(E)_{=0}=I(E) \cap K\left[X_{1}, \ldots, X_{k}\right]$ which is, by definition, the maximum of the Krull dimensions of the prime minimal ideals of $K\left[X_{1}, \ldots, X_{k}\right]$ containing $I(E)_{=0}$.

Corollary 2.3. A definable $\lambda$-closed set has a finite number of irreducible components.

Proof. A definable $\lambda$-closed set is of the form $V(J)$, where $J$ is a separable ideal of $K\left[X_{\leq n}\right]$ for some integer $n$ and $J$ contains $I^{0}(X)_{\leq n}$. The proof runs simultaneously, for fixed $n$, for all such $J \in K\left[X_{\leq n}\right]$, by induction on $\operatorname{trdg}\left(V(J)_{\leq n}\right)$. As $J$ is separable, hence radical, it is the intersection of finitely many prime separable ideals, and we can without loss of generality suppose that $J$ itself is prime. Then $\operatorname{trd} g\left(V(J)_{\leq n}\right)$ is equal to the Krull dimension of $J$.

To begin the induction, note that if $J$ has dimension 0 , then $V(J)$ is finite.

Now suppose that $\operatorname{trdg}\left(V(J)_{\leq n}\right)>0$. Applying Proposition 2.2 to $J$ produces a type ideal $Q$ and a polynomial $D \in K\left[X_{\leq n}\right]$ which are such that

$$
V(J)=(V(J) \backslash V(D)) \cup(V(J) \cap V(D))=V(Q) \cup V(J, D) .
$$

Now $V(Q)$ is irreducible and by induction, $V(J, D)$ has a finite number of irreducible components: indeed $\operatorname{trdg}\left(V(J, D)_{\leq n}\right)$ is equal to the dimension of the ideal $R:=$ $I\left(V(J, D)_{\leq n}\right) \cap K\left[X_{\leq n}\right]$. As $R$ contains the ideal $(J, D)$, and $J$ does not contain $D$, the dimension of $R$ will be strictly smaller than the dimension of $J$. 
Corollary 2.3 can be stated in the following equivalent way : there are only finitely many minimal type ideals containing any given finitely generated ideal of $K\left[X_{\infty}\right]$.

Corollary 2.4. Let $E \subseteq L^{\times k}$ be a non-empty definable set with parameters from $K$. Then there are finitely many complete types over $K, q_{1}, \ldots, q_{r}$, such that for all $i, 1 \leq i \leq r, q_{i} \vdash x \in E$ and the ideal associated to $q_{i}, I\left(q_{i}\right)$, is minimal amongst the ideals associated to complete types implying " $x \in E$ ".

Proof. Let $F(x)$ be the formula defining $E$. By quantifier elimination, $F(x) \leftrightarrow$ $\bigvee_{j=1}^{n} F_{j}(x)$ with $F_{j}(x)=\left(f_{j}(x)=0 \wedge g_{j}(x) \neq 0\right)$, for some $g_{j}, f_{j} \in K\left[X_{\infty}\right]$. By 2.3 there are types $\left(q_{j k}\right)_{k=1, \ldots, r_{j}}$ such that

$$
V\left(f_{j}\right)=\bigcup_{k=1}^{r_{j}} V\left(I\left(q_{j k}\right)\right) .
$$

Remove the $\left(q_{j k}\right)$ 's for which $g_{j} \in I\left(q_{j k}\right)$. The formula $F(x)$ belongs to each of the remaining $q_{j k}$ 's. Conversely for any $a \in L$ satisfying $F(a)$, then $a$ must be in one of these remaining $V\left(I\left(q_{j k}\right)\right)$ 's, for some $j$, that is $I(a)$ contains one of the $I\left(q_{j k}\right)$ 's.

The minimality of ideals is not in general preserved by definable bijection unless we have some extra assumptions (see section 5 for counterexamples). The birationality of the bijection is one such assumption and the next lemma is straightforward.

Lemma 2.5. Let $J \subseteq K[X]$ be a prime separable ideal. Let $q$ be the complete type over $K$ in the theory of the algebraically closed field $\bar{L}$ with associated ideal $I_{a c f}(q)=J$. Let $f$ be an injective birational map of $\bar{L}$, defined over $K$, whose domain of definition contains the realizations of the type $q$.

- Let $t$ denote the complete (acf)-type of $f(a)$ over $K$ for some (any) a realizing q. Then $I_{a c f}(t)$ is a prime separable ideal of $K[X]$.

- Let $\tilde{q}$ be the complete type in the theory of the separably closed field $L$ associated to the ideal Min $(J)$, the minimal closure of $J$. Let $r$ denote the complete (scf)-type of $f(a)$ over $K$ for some (any) $a \in L$ realizing $\tilde{q}$. Then $I(r)$ is the minimal closure of the ideal $I_{a c f}(t)$ in $K\left[X_{\infty}\right]$.

\section{Groups AND LEVEls of Rationality}

In this section, as in the rest of the paper, we use the word connected in the sense of stable groups (section 1.3).

3.1. Levels of rationality and generic types. As we mentioned in the introduction, the issue of the minimality of the ideal associated to the generic types of a definable group is at the heart of the difficulties encountered when trying to define on it a structure of algebraic group. The various levels at which the group law becomes rational turn out to be closely linked to this question, as both the positive results (Lemma 3.3 and Proposition 4.5) and the counterexamples (section 5 ) show.

Definition. Let $(G,$.$) be an M-definable group in L$, definable with parameters in $K$.

1. We say that multiplication in $G$ is rational if for all $a, b \in G, a . b \in K(a, b)$. 
In this case, by compactness, there are rational functions with coefficients in $K$, $R_{1}(x, y), \ldots, R_{n}(x, y)$, such that for all $x, y \in G, \bigvee_{1 \leq i \leq n} x . y=R_{i}(x, y)$.

2. We say that $(G,$.$) is generically rational if for all a, b$ realizing the principal generic type of $G$ and independent over $K, a . b \in K(a, b)$ and $a^{-1} \in K(a)$ or equivalently, there are rational functions $R(x, y)$ and $S(x)$, with coefficients in $K$, such that, for any such $a, b, a . b=R(a, b)$ and $a^{-1}=S(a)$.

3. Let $(G,$.$) be a definable group in L$, definable with parameters from $K$. We say that $G$ is definable at level 0 if the set $G$ is defined by a quantifier free formula in the pure language of fields.

4. Let $G$ be a definable group with principal generic type $q$. We say that $G$ is generically rational at level 0 if $G$ is definable at level 0 and if there are rational functions, $R(x, y)$ and $S(x)$, such that $a . b=R(a, b)$ and $a^{-1}=S(a)$ whenever $(a, b)$ has the same (acf)-type as the principal generic type of $G \times G$.

The following lemma is similar to Proposition 2.4 in [Me 94, where it is shown that a definable connected group is definably isomorphic to a group where multiplication is generically rational. Our more general assumptions that the group $G$ is M-definable and not necessarily connected, and the extra requirement that inverse be also rational, do not really modify the proof.

Lemma 3.1. Let $(G,$.$) be an \mathrm{X}$-definable group (definable with parameters from $K)$ in $L$. Then $G$ is definably isomorphic to a generically rational group $(H, *)$. Furthermore, if $a, b \in H$ are any independent realizations of generics of $H$ over $K$, then $a * b \in K(a, b)$ and $a^{-1} \in K(a)$.

Proof. Recall that the definable closure of $K \cup\{a\}$ in $L$ is equal to the field $K\left(a_{\infty}\right)$. It follows by compactness that there is some $n$ and there are some rational functions over $K, R_{1}\left(x_{\leq n}, y_{\leq n}\right), \ldots, R_{k}\left(x_{\leq n}, y_{\leq n}\right), S_{1}\left(x_{\leq n}\right), \ldots, S_{j}\left(x_{\leq n}\right)$, such that, if $a, b \in$ $G$ then

$$
\bigvee_{i=1}^{k} a \cdot b=R_{i}\left(a_{\leq n}, b_{\leq n}\right) \text { and } \bigvee_{i=1}^{j} a^{-1}=S_{j}\left(a_{\leq n}\right)
$$

Let $q$ be the principal generic type of the group $G$, let $g$ be a realization of $q$ and let $K((g))$ denote the following extension of $K$ :

$$
K((g))=K\left(\left\{a . g . b, a . g^{-1} . b ; a, b \in K\right\}\right) .
$$

By the above, $K((g)) \subseteq K\left(g_{\leq m}\right)$, for $m=3 n$ and must therefore be itself finitely generated, i.e. $K((g))=K\left(e_{1}, \ldots, e_{r}\right)$ for some rational functions $e_{i}=l_{i}\left(g_{\leq m}\right)$.

Let $l$ be the map defined by $l(x)=\left(x, l_{1}\left(x_{\leq m}\right), \ldots, l_{r}\left(x_{\leq m}\right)\right)$ from $G$ to $L^{\times(r+1)}$ where one replaces $l_{i}\left(x_{\leq m}\right)$ by 0 if it is not defined. The map $l$ is of course injective and one obtains by transfer a group $(H, *)$ on the image of $G$.

For any $g$ realizing $q, K(l(g))=K((g))=K\left(\left(g^{-1}\right)\right)=K\left(l\left(g^{-1}\right)\right)=K\left(l(g)^{-1}\right)$. So, if $h$ realizes $q^{\prime}=l(q)$, the principal generic type of $H$, then, $K(h)=K\left(h^{-1}\right)$. We also have that for all $a, b \in H(K), K(h)=K(a * h * b)$.

Let $K_{0}$ be a countable submodel of $K$, containing the parameters necessary to define $G$ and all the rational functions above. We now argue exactly as in [Me 94].

Let $h, b$ be two realizations of the principal generic of $H$ (over $K_{0}$ ), with $h$ generic over $K$ and with $b \in H(K)$. Then $h * b \in K(h) \cap K_{0}\left(h_{\infty}, b_{\infty}\right)$. From the independence of $h$ and $K$ over $K_{0}$, it follows that $K(h)$ and $K_{0}\left(h_{\infty}, b_{\infty}\right)$ are linearly disjoint over $K_{0}\left(h, b_{\infty}\right)$, hence that $h * b \in K_{0}\left(h, b_{\infty}\right)$. 
Let now $h^{\prime}$ be any realization of the principal generic of $H$, independent from $h$ over $K_{0}$, i.e. such that $K_{0}\left(h_{\infty}\right)$ and $K_{0}\left(h_{\infty}^{\prime}\right)$ are linearly disjoint over $K_{0}$. It follows that $K_{0}\left(h, h_{\infty}^{\prime}\right)$ and $K_{0}\left(h^{\prime}, h_{\infty}\right)$ are linearly disjoint over $K_{0}\left(h, h^{\prime}\right)$. Now $\left(h, h^{\prime}\right),\left(h^{\prime}, h\right)$ and $(h, b)$ all realize the same type over $K_{0}$, this implies that $h * h^{\prime} \in$ $K_{0}\left(h, h_{\infty}^{\prime}\right) \cap K_{0}\left(h^{\prime}, h_{\infty}\right)=K_{0}\left(h, h^{\prime}\right)$.

To generalize to all generic types, consider in $H$ two independent realizations of any generic types over $K, x^{\prime}$ and $y^{\prime}$. By saturation of $K$, all generics are congruent modulo $H^{0}$ the connected component of $H$, to some elements of $K$, hence there are $a, b \in H(K)$ such that $x^{\prime}=a * h^{\prime}$ and $y^{\prime}=g^{\prime} * b$ with $h^{\prime}$ and $g^{\prime}$ realizing the principal generic. Now $K\left(x^{\prime}\right)=K\left(a * h^{\prime}\right)=K\left(h^{\prime}\right)$ and similarly $K\left(y^{\prime}\right)=K\left(g^{\prime}\right)$, as was shown above $K\left(h^{\prime} * g^{\prime}\right) \subseteq K\left(h^{\prime}, g^{\prime}\right)$ and hence $K\left(x^{\prime} * y^{\prime}\right)=K\left(a *\left(h^{\prime} * g^{\prime}\right) * b\right)=$ $K\left(h^{\prime} * g^{\prime}\right) \subseteq K\left(x^{\prime}, y^{\prime}\right)$. Similarly, $K\left(x^{\prime}\right)=K\left(\left(x^{\prime}\right)^{-1}\right)$.

Lemma 3.2. Any definable group is definably isomorphic to a group which is generically rational at level 0 .

Proof. Let $(G,$.$) be a definable group. By Lemma [3.1, we can suppose that G$ is generically rational and by considering $\lambda_{m}(G)$ for some big enough $m$ that $G$ is definable at level 0 . Let $q$ denote the principal generic of $G$ and $q \otimes q$ the principal generic type of $G \times G$, that is the (complete) type of some (any) two independent realizations of $q$. By generic rationality, there are a rational function $R(x, y)$ such that if $(a, b)$ realize the complete type $q \otimes q$, then $a . b=R(a, b)$ and a rational function $S(x)$ such that if $a$ realizes $q$, then $a^{-1}=S(a)$.

By compactness, there is a finite part $F$ of the type $q \otimes q$ such that if $F(a, b)$ holds, then $a, b \in G, a^{-1}=S(a)$ and $a . b=R(a, b)$. By quantifier elimination, there are some integer $N$, some $f_{1}, \ldots, f_{r} \in I(q \otimes q) \cap K\left[X_{\leq N}, Y_{\leq N}\right]$ and some $g_{1}, \ldots, g_{s} \in K\left[X_{\leq N}, Y_{\leq N}\right] \backslash I(q \otimes q)$ such that $f_{1}(x)=0 \wedge \ldots \wedge f_{r}(x)=0 \wedge g_{1}(x) \neq$ $0 \wedge \ldots \wedge g_{s}(x) \neq 0$ implies that $x \in G \wedge y \in G \wedge x . y=R(x, y) \wedge x^{-1}=S(x)$. In particular, if $a, b$ is such that $I(a, b) \cap K\left[X_{\leq N}, Y_{\leq N}\right]=I(q \otimes q) \cap K\left[X_{\leq N}, Y_{\leq N}\right]$, then $a . b=R(a, b)$ and $a^{-1}=S(a)$. Take $G^{\prime}=\lambda_{N}(G)$ and transport the operation, i.e. define $\lambda_{N}(x) \cdot \lambda_{N}(y)=\lambda_{N}(x . y)$. Then $G^{\prime}$ is generically rational at level $0\left(\lambda_{N}\right.$ preserves rationality, see 1.1).

Lemma 3.3. Let $G$ be an $\mathrm{M}$-definable group in $L$, definable with parameters from $K$, with rational multiplication. Then if $q$ is a generic type of $G$, the ideal $I(q)$ is minimal amongst the prime separable ideals of $K\left[X_{\infty}\right]$ associated to complete types $r$ such that $r(x) \vdash x \in G$.

If furthermore $G$ is definable at level 0 , then $I(q)$ is the minimal closure of $I(q) \cap K[X]$ in $K\left[X_{\infty}\right]$.

Proof. Let $\psi$ be any generic formula in $G$, definable with parameters in $K$. Then there are $a_{1}, \ldots, a_{m} \in G(K)$ such that

$$
G=a_{1} . \psi \cup \ldots \cup a_{m} \cdot \psi .
$$

By rationality of the multiplication, for every $i, \operatorname{trd} g\left(a_{i} \cdot \psi\right)=\operatorname{trdg}(\psi)=\operatorname{trdg}(G)$. Similarly, as rationality is preserved by the maps $\lambda_{n}$, for every $n, \operatorname{trdg}\left(\psi_{\leq n}\right)=$ $\operatorname{trd} g\left(G_{\leq n}\right)$. If $q$ is generic, it contains only generic formulas, hence for every $n$, $\operatorname{trdg}\left(q_{\leq n}\right)=\operatorname{trd} g\left(G_{\leq n}\right)$.

If there is some $g \in G$ such that $I(g) \subset I(q)$, then for some $n, I(g) \cap K\left[X_{\leq n}\right] \subset$ $I(q) \cap K\left[X_{\leq n}\right] ;$ but this would imply that $\operatorname{trd} g\left(G_{\leq n}\right) \geq \operatorname{trdg}\left(g_{\leq n}\right)>\operatorname{trdg}\left(q_{\leq n}\right)$. 
If $G$ is definable at level 0 and if $a \in L$ is such that $I(a) \cap K[X]=I(q) \cap K[X]$, then $a \in G$. It follows directly by the minimality of the ideals associated to generic types, which is shown above, that $I(q)$ must be the minimal closure of $I(q) \cap K[X]$.

Remark. As we will see in 4.5 further down, it is enough for a definable group $G$ to be generically rational at level 0 in order to get that the ideal of the principal generic type is the minimal closure of its trace on $K[X]$. But one should beware that, even for a connected group, generic rationality at level 0 is not enough to ensure that the ideal of the generic is really minimal amongst all the ideals associated to elements of the group (see Example 5.2).

3.2. $\lambda_{0}$-algebraic groups. We will see in the next section that any infinitely definable group definably embeds into the group of $L$-rational points of an algebraic group. The natural class of groups to consider is hence the class of infinitely definable subgroups of the groups of $L$-rational points of algebraic groups. We will in fact consider here subgroups of an a priori larger class of groups, the $\lambda_{0}$-algebraic groups, which also appeared in Me 94 (under the name $L$-algebraic groups).

For $n \in \mathbb{N}$, we define the $\lambda_{n}$-topology on the affine space $L^{\times k}$ to be the (Noetherian) topology for which the closed sets are the $V(I)$ 's with $I \subseteq K\left[X_{=n}\right]$ (recall that $X_{=n}$ denotes $\left.\left(X_{\sigma}\right)_{\sigma \in\left(p^{\nu}\right)^{n}}\right)$. In particular the $\lambda_{0}$-closed sets are the Zariski closed subsets of $L^{\times k}$. The (non-Noetherian) $\lambda$-topology defined in the preliminaries is the limit of the $\lambda_{n}$-topologies.

A $\lambda_{0}$-variety defined over $K$ is a set $B=\bigcup_{i=1}^{r} B_{i}$ equipped with bijections $f_{i}: B_{i} \mapsto A_{i}, i=1, \ldots, r$, where, for $i \neq j \in\{1, \ldots, r\}$,

- each $A_{i}$ is a $\lambda_{0}$-closed subset of some $L^{\times k_{i}}$

- $A_{i j}:=f_{i}\left(B_{i} \cap B_{j}\right)$ is a $\lambda_{0}$-open subset of $A_{i}$

- $f_{i j}:=f_{j} \circ f_{i}^{-1}: A_{i j} \mapsto A_{j i}$ is rational over $K$.

The $\lambda_{n}$-topology (resp. $\lambda$-topology) on $B$ is obtained by gluing together the corresponding topologies on the affine charts.

Similarly to the classical case:

- the product of two $\lambda_{0}$-varieties is canonically a $\lambda_{0}$-variety,

- we call rational map, or morphism, a map $f: B_{1} \mapsto B_{2}$ between two $\lambda_{0}$-varieties which is rational in each affine chart,

- we define a $\lambda_{0}$-algebraic group to be a $\lambda_{0}$-variety equipped with a group structure in which both composition and inverse are morphisms.

As in the case of varieties over algebraically closed fields, we can view $B$, with its given system of finite affine charts, as a definable set in $L$. The $\lambda_{n}$-closed subsets of $B$ are definable subsets and the $\lambda$-closed subsets are $M$-definable subsets. A subset of $B$ is definable iff it is, for some integer $n$, a Boolean combination of $\lambda_{n}$-closed subsets (we say it is $\lambda_{n}$-constructible). The group law of a $\lambda_{0}$-algebraic group is of course rational in the sense of the present section.

Fact 3.4. 1. A definable $\lambda$-closed subset (or equivalently a $\lambda_{n}$-closed subset for some integer $n) C$ of a $\lambda_{0}$-variety $B$ has a finite number of $\lambda$-irreducible components.

2. A subgroup of a $\lambda_{0}$-algebraic group which is a boolean combination of $\lambda_{n^{-}}$ closed subsets (i.e. " $\lambda_{n}$-constructible") is $\lambda_{n}$-closed.

3. An M-definable subgroup of a $\lambda_{0}$-algebraic group is $\lambda$-closed.

Proof. 1. Follows from 2.3 
2. See [Me 94].

3. By stability, an M-definable subgroup is a decreasing intersection of definable subgroups. By quantifier elimination these groups are each $\lambda_{n}$-constructible for some $n$, hence $\lambda_{n}$-closed.

Proposition 3.5. Let $G$ be a $\lambda$-closed subgroup of a $\lambda_{0}$-algebraic group. Then $G$ is $\lambda$-irreducible iff it is connected (i.e. it has no proper definable subgroup of finite index) and in that case the generic type for the group law is the topological generic.

Proof. (1) If $G$ is $\lambda$-irreducible its topological generics are all in any affine chart and have all the same type, which is by 3.3 the unique candidate for being the generic in the sense of the stable group.

(2) Suppose first that $G$ is a definable subgroup. By 2.3 it has finitely many $\lambda$-irreducible components. As in the classical proof, (see for example [Hu 87], p. 53 ), only one of them, say $G_{0}$, contains the identity and $G_{0}$ is a subgroup of $G$. By (1) $G_{0}$ is connected and, as it is of finite index in $G$, it must be its connected component.

(3) Suppose now that $G$ is $\lambda$-closed and connected. It is then a decreasing intersection of definable subgroups $G_{n}$. Let $G_{n}^{0}$ be the connected component of $G_{n}$. Then $G_{n+1} /\left(G_{n}^{0} \cap G_{n+1}\right)$ is finite and $G_{n}^{0} \cap G_{n+1}$ contains $G_{n+1}^{0}$. Hence $C:=\bigcap G_{n}^{0}$ is irreducible as a decreasing intersection of irreducibles (we use here the fact that the $\lambda$-topology is a limit of Noetherian topologies). Now each $G_{n}^{0} \cap G$ has finite index in $G$ hence by connectedness of $G, G_{n}^{0} \cap G=G=C$.

\section{Definable groups And algebraic groups}

We first show (section 4.1) that a connected infinitely definable group embeds into the group of $L$-rational points of a connected algebraic group defined over $L$ and that a connected definable group is definably isomorphic to the group of $L$-rational points of a connected algebraic group ("connected" still refers to the model-theoretic notion from section 1.3 but in the case of algebraic groups this coincides with the classical notion). Then in section 4.2 we show that all definable groups are connected-by-finite. Finally in section 4.3 we generalize the result about definable connected groups to all definable groups.

4.1. The connected case. The two main tools used here will be the $\Lambda$ functor (section 1.2.4) and Weil's theorem on pregroups:

Theorem 4.1 (We 55], pages 357 and 375). Let $V$ be an irreducible variety defined over some field $k$. Let $f$ be a rational function from $V \times V$ to $V$ which is defined over $k$ and satisfies the following two conditions:

(i) If $x, y$ are generic points of $V$, independent over $k$, then $k(x, y)=k(y, f(x, y))$ $=k(x, f(x, y))$.

(ii) If $x, y, z$ are generic points of $V$, independent over $k$, then $f(f(x, y), z)=$ $f(x, f(y, z))$.

Then there is a connected algebraic group $(H,$.$) , defined over k$, and a birational map $h$ from $V$ to $H$ defined over $k$, such that, for $x, y \in V$ generic and independent over $k, h(f(x, y))=h(x) . h(y)$.

Proposition 4.2. Let $(G,$.$) be a connected \mathrm{M}$-definable group, definable with parameters from $K$. Then there is a connected algebraic group $H$, defined over $K$, such that $G$ is definably isomorphic to a subgroup of $H(L)$. 
Proof. By Lemma 3.1, we can suppose that $G$ is generically rational. Let $r$ be the generic type of $G$ (over $K$ ) and let $J$ denote $I(r) \cap K[X]$. The ideal $J$ is prime and separable. Now consider $V=V(J)$ in the algebraically closed field $\bar{L}$. This is an irreducible affine variety defined over the field $K$ with generic the (acf)-type associated to the prime ideal $J$.

The rational functions $\cdot{ }_{\mid r \otimes r}$ (where as usual $r \otimes r$ denotes the complete type of some (any) two independent realizations of $r$ ) and ${ }^{-1}{ }_{\mid r}$ give rise to a rational mapping $\circ$ from $V \times V$ into $V$, defined over $K$, which satisfies the three following conditions:

1. If $x, y$ are independent generic points of $V$ over $K$, and $z=x \circ y$, then $K(x, y)=K(x, z)=K(z, y)$.

2. If $x, y, z$ are independent generic points of $V$ over $K$, then $(x \circ(y \circ z))=$ $((x \circ y) \circ z)$.

3. If $x, y$ are two generic points of $V$ in $V(L)$ which both realize $r$ in $L$ and are (scf)-independent over $K$, then $x \circ y=x . y$.

Conditions 1 and 2 exactly mean that $(V, \circ)$ is a pregroup and that we can apply Weil's theorem (4.1). Let $H$ and $h$ be the algebraic group and the birational correspondence we obtain.

Consider $h(r)=r^{\prime}$, the image of the complete (scf)-type $r$ (in $L$ ) by $h$. As $h$ is birational and defined over $K, h_{\uparrow L}$ is an injective partial map defined with parameters in $K$ and $r^{\prime}$ is a complete (scf)-type over $K$. As $h$ respects the generic group law on $r, r^{\prime}$ is closed under generic multiplication. By Lemma 1.6, $r^{\prime}$ is the generic of a connected M-definable subgroup $G^{\prime}$ of $H(L)$ and by lemma $1.5 h$ extends to a definable isomorphism of the two connected groups $G$ and $G^{\prime}$.

In order to conclude that in the definable case, a connected group is isomorphic to the group of $L$-rational points of an algebraic group one can either use the $\Lambda$ functor, as we do now, or simply use the minimality of the ideal associated to the group generic in a group generically rational at level 0 (see Proposition 4.5 and Remark 4.7).

Proposition 4.3. Every definable connected group, definable with parameters in $K$, is definably isomorphic to the group of L-rational points of a connected algebraic group defined over $K$.

Proof. By 4.2 there is a $K$-definable embedding $f$ of $G$ into the group $H(L)$, for $H$ an algebraic group defined over $K$. Its range $f(G)$ is a definable subgroup of $H(L)$, hence $\lambda_{n}$-closed for some $n$ (3.4). Then $\lambda_{n}(f(G))$ is $\lambda_{0}$-closed in $\left(\Lambda_{n} H\right)(L)$. If $H_{1}$ is the Zariski closure of $\lambda_{n}(f(G))$ in $\Lambda_{n} H, H_{1}$ is an algebraic group defined over $K$ such that $H_{1}(L)=\lambda_{n}(f(G))$ and by density, $H_{1}$ is connected. Then $G$ and $H_{1}(L)$ are isomorphic via $\lambda_{n} \circ f$.

4.2. Definable groups are connected-by-finite. The first lemma says that irreducibility is preserved for algebraic groups (defined over $L$ ) when going down to the group of $L$-rational points. This is not true for an arbitrary variety (see section 5. This preservation result was already proved differently in Hr 96, Lemma 2.18] (using the properties of the $\Lambda_{N}$ functors in the case of groups), but our proof yields information about the generic which will be useful later on. 
Lemma 4.4. Let $H$ be a connected algebraic group defined over $K$ with (acf)generic type $r$. Then $H(L)$, as a definable group in $L$, remains connected and its (scf)-generic type, $q$, is such that $I(q)$ is the minimal closure of $I_{a c f}(r)$.

Proof of the lemma. Let $q$ be any generic type of the group $H(L)$, and let $q_{0}$ be the (acf)-type over $K$ of some (any) realization of $q$. Then the stabilizer of the type $q_{0}$ in $H(\bar{L})$ contains the group $S:=S t a b(q)$, the stabilizer of $q$ in $H(L)$, and hence also contains its Zariski closure in $H(\bar{L}), \bar{S}$. As $q$ is generic, $S$ has bounded index in $H(L)$, hence so does $\bar{S}(L)$. But $\bar{S}(L)$ is definable and by compactness must have finite index. By density of $H(L)$ in $H(\bar{L}), \bar{S}$ also has finite index in $H(\bar{L})$. By connectedness of $H, \bar{S}=H$, and the type $q_{0}$ is stabilized by the whole group $H(\bar{L})$, hence is its (unique) generic type.

The group $H(L)$ satisfies the assumptions of Lemma 3.3. Hence $I(q)$ is the minimal closure of $I(q) \cap K[X]$ which is always equal to $I(r)$ and this shows that $H(L)$ must have a unique generic.

The proof of the next result, the fact that definable groups are connected-byfinite, uses Proposition 4.2. i.e. the embedding of any connected infinitely definable group into the group of $L$-rational points of an algebraic group.

Proposition 4.5. Let $(G,$.$) be a definable group, definable with parameters from K$ and generically rational at level 0 . Let $q$ denote the principal generic type of $G$, then $I(q)$ is the minimal closure of $I(q) \cap K[X]$. Furthermore $G$ is connected-by-finite.

Proof. Consider the connected component of $G, G^{0}$ an a priori M-definable connected group with generic type $q$. Let $V=V(I(q) \cap K[X])$ in $\bar{L}$, let $R(x, y)$ be the rational function giving multiplication for independent realizations of $q$ and $S(x)$ the rational function giving the inverse on realizations of $q$. Then $V$ and $R(x, y)$ satisfy the assumptions in Theorem 4.1. Let $H$ and $h$ be the algebraic group and the birational map we obtain.

If $(a, b) \in L \times L$ are such that $I(a, b) \cap K[X, Y]=I(q \otimes q) \cap K[X, Y]$, then they are independent generics of $V$ over $K$. As $G$ is generically rational at level 0 , then also $a, b \in G$ and $a . b=R(a, b)$. It follows that $h(a \cdot b)=h(a) \cdot h(b)$.

Let $r$ be the complete (scf)-type over $K$ with ideal $I(r)$ equal to the minimal closure of $I(q) \cap K[X]$. As $G$ is definable at level $0, r \vdash x \in G$. Then,

- $h(r)$ is the generic type of $H(L)$ : by Lemma $4.4 H(L)$ is connected and its unique generic type is the (scf)-type associated to the minimal closure of $I(h(q)) \cap$ $K[X]$. By Lemma 2.5 $h(r)$ must be this generic type.

- $r$ is generic in $G$ because the stabilizer of $r$ in $G$ contains the type $q$ which is itself generic: let $a \in L$ realize $q$ and let $b \in L$ realize $r$, with $a$ and $b$ (scf)independent over $K$, then $I_{a c f}(a, b)=I(q \otimes q) \cap K[X, Y]$ and $h(a \cdot b)=h(a) \cdot h(b)$ is generic in $H(L)$, hence realizes $h(r)$.

- $r$ is stable under generic multiplication: this is true of $h(r)$ which is the unique generic of $H(L)$ and as $h$ respects generic multiplication on $r$, it must also be true of $r$.

This means that $r$ is the principal generic of $G$, and hence that $r=q$.

Furthermore, as $h(q)$ is the unique generic type of the definable group $H(L), h$ extends to a definable isomorphism between $G^{0}$ and $H(L)$ by Lemma 1.5 It follows that $G^{0}$ is in fact definable and hence that $G$ is connected-by-finite (the connected component has bounded index in $G$, if it is definable it must have finite index). 
Starting from any definable group, one can get a definably isomorphic group which is generically rational at level 0 (3.2). The next corollary follows immediately.

Corollary 4.6. Every definable group is connected-by-finite.

Remark 4.7. One can also deduce Proposition 4.3 from Proposition 4.5 without using the $\Lambda_{n}$ 's: Let $G$ be a definable connected group over $K$, by Lemma 3.2 we can suppose that $G$ is generically rational at level 0 . It follows in particular that the generic type $q$ of $G$ is the minimal closure of $I(q) \cap K[X]$. By Proposition $4.2 G$ is definably isomorphic to a subgroup of $H(L)$ for some connected algebraic group $H$, defined over $K$ and such that $H$ is birationally equivalent to $V(I(q) \cap K[X])$. It follows by Lemmas 2.5 and 4.4 that the generic of $H(L)$ must be the image of the type $q$ and hence by 1.5 that $G$ and $H(L)$ are definably isomorphic.

The next corollary applies in particular to connected $\lambda_{0}$-algebraic groups.

Corollary 4.8. If $G$ is a connected definable group, with parameters from $K$, definable at level 0 and with rational multiplication and inverse, then for some connected algebraic group $H$ defined over $L$, there is a birational definable isomorphism $f$ from $G$ onto $H(L)$, i.e. such that for every $a \in G, K(a)=K(f(a))$.

Proof. The above alternate proof of 4.3 gives directly, as $G$ is certainly generically rational at level 0 , that there is a definable isomorphism $f$ of $G$ onto $H(L)$ for some algebraic group $H$ defined over $K$, where $f$ is generically birational, that is, if $a$ realizes a generic type in $G$, then $K(a)=K(f(a))$. But a priori for $g$ non-generic, we have only that $f(g) \in K\left(g_{\infty}\right)$. Now if $g$ is any element of $G$ in $L$, pick $a$ generic in $G$ over $K$ and independent from $g$ over $K$. Then $f(g)=f(g a) \cdot f\left(a^{-1}\right) \in K(g, a)$, as both $(g a)$ and $a^{-1}$ are generics. Hence $f(g) \in K(g, a) \cap K\left(g_{\infty}\right)$. By independence of $g$ and $a$ over $K, K\left(g_{\infty}\right)$ and $K\left(a_{\infty}\right)$ are linearly disjoint over $K$. It follows that $K(g, a)$ and $K\left(g_{\infty}\right)$ must be linearly disjoint over $K(g)$, hence that $f(g) \in K(g)$.

4.3. The general case. We know now that definable groups are connected-byfinite (4.6) and that connected definable groups are definably isomorphic to the group of $L$-rational points of a connected algebraic group (4.3). The argument given in the next proposition, in order to show that all definable groups are definably isomorphic to the group of $L$-rational points of some algebraic group defined over $K$, is quite similar to the argument given in [Po 87, 4.e] for constructible groups in an algebraically closed field. There is though an additional step needed at the end, which requires the use of the $\Lambda_{n}$ 's introduced in section 1.2.4

Proposition 4.9. Let $G$ be a definable group, definable with parameters from $K$, then there is an algebraic group $H$ defined over $K$ such that $G$ is definably isomorphic to $H(L)$.

Proof. By 4.6 $G$ is connected-by-finite; let $G^{0}$ be its connected component, $\widetilde{G}=$ $G / G^{0}=\left\{c_{1}, \ldots, c_{r}\right\}$. Let $R=\left\{a_{1}, \ldots, a_{r}\right\}, a_{j} \in c_{j}$, be a set of representatives for $\widetilde{G}$ chosen so that $a_{1}=1$ and $\left(a_{j}\right)^{-1}=a_{k}$ when $c_{k}=\left(c_{j}\right)^{-1}$. Let $d(i, j) \in G^{0}$, for $1 \leq i, j \leq r$, be such that $a_{i} \cdot a_{j}=a_{i . j} . d(i, j)$. Then $G$ is definably isomorphic to $\widetilde{G} \times G^{0}$ endowed with the operation

$$
\left(c_{i}, g\right) \cdot\left(c_{j}, g^{\prime}\right)=\left(\left(c_{i} \cdot c_{j}\right),\left(d(i, j) \cdot g^{a_{j}} \cdot g^{\prime}\right)\right) .
$$

By 4.3, there is a connected algebraic group $H$, defined over $K$, and a $K$-definable isomorphism $h$ from $G^{0}$ onto $H(L)$. Let $e(i, j)=h(d(i, j))$ and let $\alpha_{i}$ be the definable automorphism of $H(L)$ corresponding to the conjugation by $a_{i}$ in $G^{0}$. 
We are going to change the group variety structure on $H$ in order to transform the definable automorphisms $\alpha_{i}$ into rational automorphisms of $H$. This will have to be done in two steps.

First let $H_{1}=H^{\times r}$ and embed the group $H(L)$ into $H_{1}(L)$ by the map $f$ : $x \mapsto\left(\alpha_{1}(x), \ldots, \alpha_{r}(x)\right)$. Then one can check that, for every $\alpha_{i}$, the corresponding automorphism of $f(H(L)), f \circ \alpha_{i} \circ f^{-1}$, is indeed the restriction of a morphism $\gamma_{i}$ of the algebraic group $H_{1}$ defined over $K: \gamma_{i}$ is a composition of permutation of coordinates and of conjugation by the $d(i, j)$ 's, which are elements of $H(K)$. But the group $f(H(L))$ is a definable subgroup of $H_{1}(L)$ which so far has no reason to be of the form $H_{2}(L)$ for some closed subgroup $H_{2}$ of $H_{1}$. This is what the next step will achieve.

For some $N \geq 1$, big enough, $\lambda_{N}(f(H(L)))$ is a subgroup of $\left(\Lambda_{N} H_{1}\right)(L)$ which is definable at level 0 and hence is equal to $H_{2}(L)$, where $H_{2}$ is its Zariski closure in $\Lambda_{N} H_{1}$. There remains to check that the morphisms $\gamma_{i}$, transported via $\Lambda_{N}$ give rise to $K$-rational automorphisms of $H_{2}$, which we leave to the reader.

Now starting from the connected algebraic group $H_{2}$, defined over $K$, the automorphisms $\Lambda_{N} \gamma_{i}$ and the points $\lambda_{N}(f(e(i, j)))$, we let $H_{3}=\widetilde{G} \times H_{2}$, with the adequate multiplication (as in $(*)$ ). Then $H_{3}$ is an algebraic group defined over $K$, with connected component isomorphic to $H_{2}$ and $G$ is definably isomorphic to $H_{3}(L)$.

Putting this together with the framework introduced in Section 3.2 , we get finally:

Proposition 4.10. Any group $\mathrm{M}$-definable in $L$, with parameters from $K$, is definably isomorphic to a $\lambda$-closed subgroup of the group of $L$-rational points of some algebraic group defined over $K$.

Proof. By stability, any infinitely definable group $G$ is a subgroup of a definable group. By Proposition 4.9. this definable group is definably isomorphic to the $L$ rational points of an algebraic group $H$ and by 3.4 the image of $G$ is a $\lambda$-closed subgroup of $H(L)$.

\section{EXAmples}

We present first an irreducible variety $V$ defined over $K$ and such that $V(L)$ is not irreducible in the $\lambda$-topology, in contrast to the case of algebraic groups (see Lemma 4.4).

Then we construct two (affine) $\lambda$-closed definable groups with generically rational group law which illustrate the non-coincidence of topological generic and group generic.

5.1. An irreducible variety $V$ such that $V(L)$ is not $\lambda$-irreducible. Let $J \subseteq K[X, Y, Z]$ be the ideal generated by the polynomial $Y^{p^{m}} X+Z^{p^{m}}$, where $X, Y, Z$ denote variables and not tuples of variables and $m \geq 1$. This polynomial is linear in $X$ and irreducible, hence the ideal $J$ is prime and separable (the fraction field of $K[X, Y, Z] / J$ is isomorphic to $K(b, c)$ for some algebraically independent $b, c)$ and defines on $\bar{L}$ an irreducible variety $V$, defined over $K$. We are going to see that the ideal $J$ is contained in exactly two distinct minimal type ideals of $K\left[X_{\infty}, Y_{\infty}, Z_{\infty}\right]$, that is that the $\lambda$-closed set $V(J) \subset L^{\times 3}$ has exactly two distinct irreducible components. This also gives a counterexample to Lemma 46 in [De 88]: the separable closure of $J+I^{0}(X)+I^{0}(Y)+I^{0}(Z)$ in $K\left[X_{\infty}, Y_{\infty}, Z_{\infty}\right]$ is not prime. 
First let us find the minimal closure of $J$, as in 2.1] Let $a, b, c \in L$ with $b$ and $c$ as above and $a=-(b / c)^{p^{m}}$. Then we can take the polynomial $D$ to be equal to $Y$ (recall that the condition on $D$ in 2.1 was that $a \in K\left[b, c, a^{p},(D(b, c))^{-1}\right]$ ). Let then $Q \subseteq K\left[X_{\infty}, Y_{\infty}, Z_{\infty}\right]$ be the unique minimal type ideal containing $J$ and not $Y$.

If any separable ideal contains $J$ and $Y$, it must contain $Z$. Let $R=I(V(Y, Z))$ ( $R$ is the separable closure of $\left(Y, Z, I^{0}(X)+I^{0}(Y)+I^{0}(Z)\right)$ ), then $R$ is prime and hence also a type ideal containing $J$. Let us see that $R$ does not contain $Q$. Indeed, any type ideal which, like $Q$, contains $Y^{p^{m}} X+Z^{p^{m}}$ and does not contain $Y$ must also contain $X_{\sigma}$ for all $\sigma \in\left(p^{\nu}\right)^{m}, \sigma \neq \underline{0}$ (recall that $M_{\underline{0}}$ is equal to 1 ). These are certainly not in $R$ which can hence not contain $Q$.

Now given any type ideal which contains $J$, either it does not contain $Y$ and must then contain $Q$, or it contains $Y$ and must then contain $R$. Consequently $Q$ and $R$ are the only two minimal type ideals containing $J$ and $V(Y, Z)$ and $V(Q)$ are the two irreducible components of $V(J)$.

5.2. A connected definable group with non-minimal ideal associated to the generic. This example is similar to the one given in $\mathrm{Po} 87,5 . c]$ to show that, in contrast to the superstable case, in a stable non superstable group, generics are not defined independently of the group law and that there can be two definable group structures with different generics defined on the same set.

Here we put another group structure on $(L,+)$. Consider the definable bijection $f$ from $L$ to $L$ defined in the following way:

- if $x \in L \backslash L^{p}, f(x)=x^{p}$,

- if $x \in L^{p} \backslash L^{p^{2}}, f(x)=x^{1 / p}$,

- if $x \in L^{p^{2}}, f(x)=x$.

Transporting addition through $f$, one gets a group on $L$ again, $G:=(L, *)$, definably isomorphic to $(L,+)$, hence connected. The set $L$ itself is of course $\lambda$ closed and irreducible with ideal $I(L)=I^{0}(X)$.

The ideal associated to the (group) generic type of $(L, *)$ is generated by $I^{0}(X)$ and $\left\{X_{i}=0 ; i \in p^{\nu}, i \neq 0\right\}$, and strictly contains $I^{0}(X)$ (and is in fact the generic of the group $\left.\left(L^{p},+\right)\right)$.

The group law is generically rational but it is not everywhere rational in the sense of section 3

By considering the group $\lambda_{2}(G)$, one gets a group structure on $L^{\times p^{2 \nu}}$ which is generically rational at level 0 and also has a generic with non-minimal ideal.

The situation here is different from algebraically closed fields. In algebraically closed fields, the topological generic of any irreducible Zariski closed set is the unique type of maximal Morley rank, hence must be the group generic of any definable group structure on it. Furthermore, in an algebraically closed field of any characteristic, if $H$ is a connected constructible group with generically rational multiplication and inverse, then $H$ is birationally equivalent to a connected algebraic group. As Lemmas 2.5 and 3.3 show, the above group $G$ cannot be birationally equivalent (in the obvious sense) to the group of $L$-rational points of a connected algebraic group.

5.3. An irreducible $\lambda$-closed group which is not connected. As in algebraically closed fields, there are definable closed connected groups which are not irreducible. We give now an example of a group defined on an irreducible $\lambda$-closed 
set which is not connected. This is impossible in the algebraically closed case: a group which is not connected has more than one type of maximal Morley rank while an irreducible closed set must have a unique type of maximal Morley rank.

In order to simplify notation, let us suppose that $p=2$ and that $\left[L: L^{2}\right]=2$. Let $b \in K \backslash K^{2}$. Consider the group

$$
G=(L,+) \times\left(\left(L^{2} \backslash\{0\}\right) \cup\left(b L^{2} \backslash\{0\}\right), \times\right) .
$$

We are going to find a definable injective map from $G$ onto a closed irreducible set. The underlying set of the group $G$ is $\left(L \times\left(L^{2} \backslash\{0\}\right)\right) \cup\left(L \times\left(b L^{2} \backslash\{0\}\right)\right)$, which is in definable bijection, using the inverse of the Frobenius, with the union of two copies of the set $(L \times(L \backslash\{0\}))=(L \times L \backslash L \times\{0\})$. This in turn is in definable bijection, through the pairing function $\lambda$ on one hand, and the canonical isomorphism of $L \times L$ onto $L \times L \times\{(0,0)\}$ on the other hand, with

$$
\begin{gathered}
(L \times L \times L \times L \backslash L \times L \times\{(0,0)\}) \cup(L \times L \times\{(0,0)\} \backslash L \times\{(0,0,0)\}) \\
=L \times L \times L \times L \backslash L \times\{(0,0,0)\} \\
=L \times(L \times L \times L \backslash\{(0,0,0)\}) .
\end{gathered}
$$

The definable injective map

$$
(x, y, z) \mapsto\left(x, y, z,\left(x^{4}+b y^{4}+b^{2} z^{4}\right)^{-1}\right)
$$

sends $(L \times L \times L \backslash\{(0,0,0)\})$ onto the irreducible closed subset $F$ of $L^{\times 4}$ given by the equation $\left(x^{4}+b y^{4}+b^{2} z^{4}\right) t=1$. The cartesian product of two irreducible closed sets is closed and irreducible, $L \times F$ satisfies our requirements.

\section{REFERENCES}

[BoDe] E. Bouscaren and F. Delon, Minimal groups in separably closed fields, to appear in the Journal of Symbolic Logic.

[De 88] F. Delon, Idéaux et types sur les corps séparablement clos, Supplément au Bulletin de la SMF, Mémoire 33, Tome 116 (1988). MR 90m:03067

[De 98] F. Delon, Separably closed fields, in Model Theory and Algebraic Geometry, E. Bouscaren (Ed.), Lecture Notes in Mathematics 1696, Springer, 1998. MR 2000a:12011

[Hu 87] J.E. Humphreys, Linear Algebraic Groups, Graduate Texts in Mathematics, Springer, 1987. MR 53:633

[Hr 96] E. Hrushovski, The Mordell-Lang conjecture for function fields, Journal AMS 9 (1996), 667-690. MR 97h:11154

[La 58] S. Lang, Introduction to algebraic geometry, Interscience Tracts in Pure and Applied Mathematics, Interscience Publishers, New York, 1958. MR 20:7021

[Me 94] M. Messmer, Groups and fields interpretable in separably closed fields, TAMS 344 (1994), 361-377. MR 95c:03086

[Me 96] M. Messmer, Some model theory of separably closed fields, in Model Theory of Fields, Lecture Notes in Logic 5, Springer, 1996. MR 98m:03075

[Pi 96] A. Pillay, Geometrical Stability Theory, Oxford University Press, 1996. MR 98a:03049

[Pi 98] A. Pillay, Model theory of algebraically closed fields, in Model Theory and Algebraic Geometry, E. Bouscaren (Ed.), Lecture Notes in Mathematics 1696, Springer, 1998. MR 2000f: 12008

[Po 87] B. Poizat, Groupes Stables, Nur al-mantiq wal ma'rifah, Villeurbanne, France, 1987. MR 89b:03056

[Sp 98] T.A. Springer, Linear algebraic groups, 2nd edition, Birkhäuser, 1998. MR 99h:20075 
[Wa 97] F. Wagner, Stable Groups, London Math. Soc. LNS 240, Cambridge University Press, 1997. MR 99g:20010

[We 55] A. Weil, On algebraic groups of transformations, American Journal of Math. 77 (1955), 355-391. MR 17:533f

Université Paris 7- CNRS, UfR de Mathématiques, Case 7012, 2 Place Jussieu, 75251 PARIS CEDEX 05, France

E-mail address: elibou@logique.jussieu.fr

Université Paris 7- CNRS, UFR de Mathématiques, Case 7012, 2 Place Jussieu, 75251 PARIS CEDEX 05, France

E-mail address: delon@logique.jussieu.fr 